Proceedings

\title{
Beyond the Arrow of Time: Can There Be a Relation between the Measurement of Entropy and Time? ${ }^{\dagger}$
}

\author{
Ben Akih-Kumgeh \\ Department of Mechanical and Aerospace Engineering, Syracuse University, NY 13244, USA; \\ bakihkum@syr.edu; Tel.: +1-315-443-2335 \\ + Presented at the 4th International Electronic Conference on Entropy and Its Applications, \\ 21 November-1 December 2017; Available online: http:/ / sciforum.net/conference/ecea-4 \\ Published: 20 November 2017
}

\begin{abstract}
The general tendency of the entropy of an isolated to increase is considered to be directly linked to the direction of the flow of time. This raises the question whether a quantitative relation can be established such that a time interval can be measured by measuring entropy change and vice versa. The existence or absence of such a link also calls for further consideration of the nature of time. Prigogine argued that the true nature of time can only be discovered by investigating this phenomenon using scientific and philosophical methods. If this is true, then ongoing debates in the metaphysics of time and progress in the scientific study of entropy can be brought together to shed light on this fascinating but elusive concept. In this paper, starting from my recent modified definition of entropy change as a non-dimensional measure of energy change, a direct link between entropy and time duration is suggested. It draws from steady energy transfer processes such as heat transfer and shows that a measure of time can be found to be associated with a measure of entropy change. In the absence of other driving forces, the passage of time in an isolated system can therefore be tracked with a well calibrated entropy change meter. When other forces are allowed to interfere and there is no external point from which the system can be considered to be isolated, then the measure of time is non-monotonous since an isolated system can be restored to an earlier state of non-equilibrium. More philosophical questions about time can be reduced to questions about the operation of memory and consciousness, whereby psychological awareness of time is in essence a mental awareness of the way things are ordered by direct perception and memory of how they were ordered in the past, permitting a measure of the departure between things changing with greater or lesser intensity.
\end{abstract}

Keywords: arrow of time; entropy; heat transfer; irreversibility; metaphysics of time

\section{Introduction}

The idea that the entropy of an isolated system only increases has been taken to indicate that time has a distinctive direction that aligns with the direction of natural processes. This is supposed to be a departure from the classical mechanical views of the basic laws of nature being reversible. Many questions arise from these startling discoveries. Curiosity demands further investigation into the nature of the relation between entropy and time. Beyond the arrow of time suggested by the natural tendency of entropy to increase in isolated systems, can there be a quantitative relation such that proper calibration would allow for the measurement of one quantity through the measurement of the other? It seems that once we are clear about the proper physical meaning of entropy as a non-dimensional measurement of the capacity to cause change and recognizing that time is intricately connected to change in the way things are in the world, such a relation can be recognized to exist. But if the relation does exist, then the fact that entropy is the measurement of actual observable changes in the physical world brought about by the hidden dispositions we call energy, raises questions about the nature of 
time itself, since it has been suggested by some philosophers that time is unreal. This is a difficult question, the answer of which might be found through investigation of consciousness and memory by whose help we come to be aware of the passage of time. But focusing on change in the way things are in the world, the reality but possibly non-uniqueness of time might be asserted, knowing fully well that any perturbation of the isolated system by forces from without or within that are not accounted for in a process, might reverse or slow down the time computed simply from observing changes in the system between two different states, with unobserved perturbation events in-between.

The irreversibility of processes in isolated thermodynamic system, hence its alignment with time asymmetry, was first met with resistance. This was motivated by a supposed greater familiarity with the reversibility that is supposed to follow from the laws of classical mechanics. But if entropy and time are to be related beyond the indication of time asymmetry, then it must be explained why time symmetry in classical mechanics obtains. It can be argued that this is based on a faulty distinction between the properties of physical systems and the properties of the mathematical language used to express properties of the physical world. This is not pursued extensively in this paper but can be elaborated.

The above discussion might be viewed with suspicion: it mixes physics with metaphysics in the discussion of physical quantities. But this is not enough ground to dismiss the topic altogether. When foundational questions are raised about scientific methods and concepts, one inevitably ends up in the domain of philosophy, where what seems to be a trivial problem rapidly drifts into the realm of the skeptical as we try to clarify the concepts in a non-circular manner. It was the view of Prigogine that greater insight into the nature of time is to be found at the interface of science and philosophy $[1,2]$. This paper is motivated by that optimistic hope of interdisciplinary enlightenment and tries to explore what positive relation might exist between entropy and time measurements. One would like to have both grounded in a common concept or phenomenon that is more fundamental. I see this more basic thing to be the concept of change in material configuration in space. Psychological time emerges as awareness of part of the cosmos (such as satient beings) about the relative motions/changes between that part and other parts of the cosmos. The grounding of time in change or causation can already be found in Aristotelian Physics [3] and the connection of entropy to change as the result of 19th century physics [4,5], illuminated by my emphasis that viewing entropy as a non-dimensional measure of energy change associated with non-equilibrium interactions of systems [6]. The paper is written from the perspective of conceptual analysis with minimal use of equations to establish a possible relationship.

\section{Analysis of Entropy Change and Time Measurements}

We first need to consider some views about time and time measurements as well as entropy change before attempting to link the two.

\subsection{Time}

If space, time, matter and change are ubiquitous and are the means by which we structure our experiences of the world [7], it seems that we are doomed to a certain circularity or guess work in trying to get at the true nature of all of these concepts. Each one of them can only be understood by declaring knowledge of some or all of the others. But philosophy has tried to get at the nature of time as a thing in itself whose properties are to be discovered. Questions on the foundations of time are to be found in metaphysics and views on the problem have been expressed from the very beginning of Greek natural philosophy. In physics, however, views on time can split between Newtonian views of time and the geometrization of time achieved by the founders of relativistic mechanics. The geometrization of time is not without controversy, since properties of the time axis presented in relativity is nothing like the other three spatial coordinates $[1,8,9]$. The unification of philosophical time and physical time as sought by Prigogine seems therefore to be made more difficult by the role of time in relativity. One must first retreat to time in classical physics to appeal to intuition in making judgments about 
any tentative theories of time and time measurements. An acquaintance with time in philosophy and physics must be factored into any exploration of time and entropy relations.

\subsubsection{Time in Philosophy}

The approach sought here to relate entropy change to time measurement might be said to have its roots in Aristotelian physics [3]. Aristotle considers change and time to be intricately related and seeks to give an account of time in terms of change, making change a more fundamental feature of the physical world. Change or causation has to do with the actualization of inherent dispositions (that is, capacities or potentialities) in things. Aristotle therefore holds that the "before" and "after" of a physical change can be translated into a "before" and "after" in time measurement. The task of this measurement then becomes one of following stages in the process of change. Change itself might be understood to be a set of yet smaller changes, thereby making possible subdivisions of time and change. Now, this change in things as we perceive might be grounded in measurements of their capacities or their material location and configuration in space. The connection between classical thermodynamics that is focused on "smearing out" and averaging macroscopic properties and the statistical mechanics of counting configurations of microscopic particles allows us to connect time to change in the way things are in the world. If these changes are natural, then they will be such that the entropic measure of change increases in the ordering of "before" and "after" states of affairs.

Aristotle's account of time might be rightly considered as being focused on an account of time measurement, rather than time as the thing in itself. What then is the actual nature of time? The question remains and calls for an account of what is to be construed as a thing, seeing that most of what we call things are actually relations of relations ... of things. For instance, we speak of rain fall whereas we mean water molecules that stand in a certain relation to space, air, gravity and other water molecules. These relations might be classed as abstract entities in contradistinction to substance, which can be supposed to consist of pure or composite particles of matter in space.

The question about the nature of time and our psychological awareness of it remains, after we are acquainted with the Aristotelian view of time being related to the before and after of given changes. Aristotelian time can be explored by focusing on the physical world alone, tracking changes in the configuration of things and relating them to number and quantity, hence the changes in the state of physical things to measures of time. A human mind does not necessarily enter directly into the investigation. Some light on the psychology of time can be gained through a reading of St. Augustine's views on the question of our experience of time as expressed in his Confessions [10]. According to Augustine when we measure the duration of an event or interval of time, is in the memory. But since the past is gone and what we perceive presently has no duration, from this might arise the radical conclusion that past and future exist only in the mind. The motion of thoughts recalling the past and comparing with the present is needed to produce the experience of the passage of time in our minds. This psychological measure of time cannot advance a physical measure of time and entropy; there is inherent subjectivity and absence of units of measurements. Apart from memory, one can also introduce imagination in which our thoughts move along imagined beginnings and endings of events or processes. The focus on psychological time without the Aristotelian notion of time being related to the before and after of change can be understood to be the reason for McTaggart's view that time is unreal [11].

If the unreality of time asserted by McTaggart is to be refuted, then the reality of time calls for an answer as to the uniqueness of time. Proceeding from the idea that instants are related by notions of before and after, Swinburne [8] asserts that all instants that are temporally related constitute a time. Of logical necessity, time is therefore unique. But since our experience of time in physical science or psychology is limited by physical resolution and complete knowledge of the whole universe, the relatedness of all relative motions and various assumptions about uniform properties cannot be established. This also limits our access to the unique time, if it exists. 
It seems therefore than the philosophical views of time cannot converge on a unique picture of time but it does furnish us with useful ideas that can be used in physical time discussions. The first is Aristotle's relation of time to change and the second is Augustine's idea of the role of memory in our awareness of time. Unconscious things that undergo change, by their changing properties do leave behind a history which is only discoverable through the ability to be aware of a before and an after. This ability calls for consciousness and memory.

\subsubsection{Time in Physics}

The view of time in Physics can be divided into time as understood in the classical physics of slowly moving systems and time in relativistic mechanics where it becomes similar to the dimensions of space.

In classical physics, Newton distinguishes between absolute and relative time, the former being unconstrained by the contingent motions in the universe by means of which time's relative measure is carried out. There is a sense in which this relative time be traced to the Aristotelian connection of durations of time to the before and after of physical processes in the world. Taking a day as a unit of time, the question of its definition arises. What is a day? This used to be viewed as the period of time it takes a fixed star such as our sun to return to zenith. This time is taken to be constant, because the celestial sphere on which the fixed stars are located can be considered to neither speed up nor slow down [12]. Kepler's work raised questions about this assumed uniformity in the motion of celestial bodies. The invention of the pendulum clock provided a way to measure uniform time on earth, assuming regularity of the gravitational field pulling on the pendulum.

Apart from this relative time perceivable through relative motion, Newton advanced the idea of absolute time which is independent of perception or processes but progresses uniformly. This absolute time therefore allows for mathematical treatment of time that is void of the prejudices of any observer. Newton's position therefore points to the existing of a unique, absolute time in addition to the relative time that is accessible to our experience. Swinburne tentatively agrees with him on grounds a true standard of simultaneous events can be formulated and a true measure of equal time intervals can be conceived [8]. This absolute time can only be approached from the conceptual framework of relative change if the first cause of the physical universe and complete knowledge of all relative motions can be brought into view.

The notions of absolute time and absolute space in classical physics have been superceded in the theories of special and general relativity in favor of a unified space-time structure. Taken together with the speed of light, time becomes a coordinate with some properties that are similar to the other three dimensions of space [8,9]. Although this innovation advances the treatment of the mathematical physics of celestial bodies, Reichenbach observes that treating time as a fourth dimension adds to the confusion of about the concept of time [9]. Instead of focusing on how time might be measured and what its properties are, the space-time treatment in relativity seems to invite the observer to visualize the extension of a dimension of time in a four dimensional presentation that is hardly intuitive.

These contentious views about absolute time and the geometrization of time, notwithstanding, relative time plays an important role in science and is measured through various motions with tacit assumptions about the uniformity of a certain aspect of the phenomenon employed. Thus, a wide range of devices and phenomena are used for time measurements, the most accurate of which is the atomic clock [13]. The atomic clock makes use of a quantized energy emission in closely lying quantum states of caesium 133 atom, which with a constant speed of light and Planck constant yield a standard frequency of 9, 192, 631, $770 \mathrm{~Hz}$. It seems then objective measures of relative time are realized through changes in other physical things in line with Aristotle's views. The question is whether the change captured by entropy change can be a candidate for such time measurement. This calls for a brief overview of entropy change. 


\subsection{Entropy Change}

In my previous work [6], I argued that specific entropy change should be viewed as a non-dimensional measure of energy change associated with non-equilibrium interaction of two systems or parts of a system in a state of non-equilibrium. The main idea was to view differential entropy as $d s=f r a c \delta q \epsilon$, where the intensive energy quantity $\epsilon$ is taken as $\epsilon=k T$. The actual entropy change is then simply a product of this non-dimensional specific entropy change and the particle number. In the case of two systems, such as a hot metal block and a colder one, their interaction is that of the ensuing non-equilibrium heat transfer upon thermal contact, even though each system is in an initial state of equilibrium, if shielded from their environment.

This view therefore established that a thermodynamic driving force must be imposed for the entropy of the system to change. In the case of a system in non-equilibrium, the properties of the system might be considered to be described by very coarse-grain averages while the system exhibits spatial or temporal non-uniformity.

For clarity, we shall adopt the heat transfer problem in which heat is transferred from a hotter body (source) to a colder one (sink). We consider the systems to be non-deformable so that all heat exchanges end up as internal energy changes. Let us further assume that heat capacities of the two blocks are infinite, so that while the energy of the hotter body is reduced and the colder one increased, the temperatures remain fairly constant and the Fourier law governing the rate of heat transfer leads a fairly constant rate of heat transfer. The two blocks are separated from the rest of the universe both in terms of energy and mass exchange, constituting a larger system that is isolated. The energy of the system, describing the internal motion of the constituting particles is therefore constant. The internal energy changes and the entropy change can then be described as in the following set of equations:

$$
\begin{gathered}
\int_{\text {isol. syst }} d E=0=>E_{\text {sink }}+E_{\text {source }}=\text { const }=E_{0} \\
\left.d E\right|_{\text {sink }}=\delta Q \\
\left.d E\right|_{\text {source }}=-\delta Q=-\left.d E\right|_{\text {sink }} \\
\Delta S=\left.\int_{1}^{2} \frac{\delta Q}{k T}\right|_{\text {sink }}+\left.\int_{1}^{2} \frac{\delta Q}{k T}\right|_{\text {source }}
\end{gathered}
$$

By replacing the heat terms in Equation (4) and considering the constant temperatures, one can establish the entropy change. The internal energy change as a function of time can be related to the integrated heat flux.

\subsection{Time and Entropy Changes}

It might be said that a relation between entropy and time is already given by the entropy production rate. De Donder's work on the rate of chemical reactions and entropy generation within a system might be seen as the foundation of non-equilibrium thermodynamics in which entropy and time comes together $[14,15]$. Entropy production is then expanded by Onsager to account for multiple driving forces and their reciprocal constraints on the rate [16]. The form of the equations and the complication introduced by multiple driving forces obscure attempts to arrive at conceptual clarity on a possible link between entropy and time measurements.

If we return to the expression for the entropy change in the heat transfer problem shown in Figure 1 and the equations previously established, we can seek a direct relation by introducing the changes in internal energy which are related to the total heat transferred as determined by integrating the heat transfer flux as in the sequence of equations that follow:

$$
\dot{Q}=-k A \frac{d T}{d x} \approx \text { const }=K
$$




$$
\int_{0}^{Q(t)} \delta Q=\int_{t_{0}}^{t} K d t=>Q(t)=K\left(t-t_{0}\right)
$$

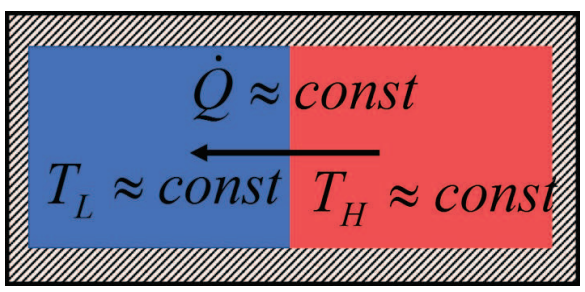

Figure 1. A schematic of an isolated system with two sub systems at equilibrium but at different temperatures so that heat flows from the hotter to the colder subsystem. The heat capacities of the two subsystems are assumed to be infinite so that the temperatures remain fairly constant, their difference therefore driving a fairly constant heat flux. This corresponds to a near equilibrium interaction for each system (linear non-equilibrium), so that the internal energy of each subsystem increases linearly with time.

The integration of the one-dimensional Fourier law yields a linear relation between the heat transferred, $Q$, within the time duration, $t-t_{0}$, with a constant of proportionality that is determined by the surface area, conductivity, and temperature gradient across the thermal contact zone. With this equation, the overall entropy change in Equation (4) can be found, taking into account the constant temperature and conservation of the total energy of two blocks. The result can be re-written as in Equation (8).

$$
\begin{gathered}
\Delta S=Q(t)\left(\left.\frac{1}{k T}\right|_{\text {sink }}-\left.\frac{1}{k T}\right|_{\text {source }}\right)=K\left(t-t_{0}\right)\left(\left.\frac{1}{k T}\right|_{\text {sink }}-\left.\frac{1}{k T}\right|_{\text {source }}\right) \\
\Delta S=\text { const. }\left(t-t_{0}\right)
\end{gathered}
$$

This equation shows that with the simplifying assumptions we have used (thereby making the problem one that is very close to the respective equilibria), the measure of entropy change is directly proportional to the measure of a time duration, with the constant of proportionality determined by the two temperatures and the fairly constant heat flux. The condition of constant temperatures can be relaxed, keeping the constant heat flux assumption. If this is done, an exponential relation between entropy change and time will ensue. If both constant heat flux and constant temperature assumptions are relaxed, a complex relation can still be obtained. The crucial idea is that for a given system where an aspect of the natural process can be assumed to be uniform, a relation between entropy change after a given time and a measure of time duration can be obtained. This consideration of one driving force that is related with entropy therefore answers in the affirmative question whether a positive relation between time duration and entropy change can be found such that the passage of time is measured from the evolution of a steady heat transfer process. The heat transfer process in this case is to be tracked by measurement of the changes in the internal energy in each subsystem. It is besides the point here that our assumption of in-deformable subsystems and fairly constant temperature would make indirect measurement of the internal energy changes of the system difficult. A practical heat transfer clock should allow for changes in thermodynamic properties from which changes in the metaphysical energy changes can be inferred.

There are a number of situations where inferring time from entropy accumulation can lead to problems. Suppose we measure the entropy of the two systems and go away. Before we come back, it can happen that additional heat is produced by a chemical reaction in one of the bodies or both. It might also be that one or both of them has lost their insulation and leaked energy to the surrounding. If the conserved energy assumption and the operation of a single driving force cannot be verified, our inference of time durations from entropy differences is therefore vulnerable to undetectable falsification. Only in the case of entropy decrease, such as excessive heat loss from either block that 
leads to overall reduction of entropy over time, would the entropy change raise an alarm. The decrease in entropy following a before and after measurement would serve as the surest sign of an external agent resetting the time and entropy. Extending this to our measurement of time, this is not entirely unthinkable since a sudden uniform change in the rate of all processes in the universe (including the operation of our memories) could go undetected by us as a general slowing down or speeding up of the passage of time.

From the relation we have established, a time rate of change of entropy would therefore be a constant, indicating the steady physical relation between the two quantities. However in Onsager's equation for the rate of entropy production inside a system, this time rate of change is determined by the fluxes leading to entropy change, $J_{p}$, and the extension in the associated generalized coordinate, $X_{p}$ :

$$
\frac{d_{i} S}{d t}=\sum_{p} J_{p} X_{p}
$$

For two reciprocal processes, measuring entropy change through thermodynamic properties of the system might be complicated by the different effects that each process has on the thermodynamic properties which can still yield the same total energy. For a single process, the rate of entropy production can be constant if the flux is constant. The measurement of time based on the measurement of entropy change must proceed by integrating Equation (9), which may not be linear or monotonous, in the case of perturbations from without, such as energy injection or withdrawal.

\section{Further Discussion}

The analysis presented above can be further discussed with respect to some implications and challenges to the proposed view. These are briefly considered here.

\subsection{Implication of the Supposed Connection}

It seems that if durations of time and entropy changes are related, in addition to the direction indicated by natural processes, then one must say something about McTaggart's famous assertion that time is unreal [11]. The question hinges on our understanding of reality. We need to answer whether mental representations of things like the computer infront of me are real. The computer can be taken to be real-it is matter in space but the representation I make of it has a different feeling of reality. If someone denies the reality of the computer, I must first seek to establish which reference is being used-the computer external to me or the computer represented in my mind. So when we say time is unreal and we are sympathetic to Aristotle's and St. Augustine's grounding of time in changes in material things, we must distinguish between our perception of changes and the changes in material things themselves.

The distinction between reality of things in space, external to us and representation of these things in our minds, is not a trivial one. It can also be brought to bear on the distinction between energy in itself as a metaphysical quantity and the effect of energy that can be observed. It is the case that energy cannot be directly measured or observed; we are contented to infer the existence of capacities in things to cause change from observing things being deformed or made to move in space. Pushed harder for a conceptual distinction between force and energy, our inability to measure these things directly, move us to adopt ways of inferring their existence from changes of matter in space. What we have established above is time duration as an awareness of relative changes in dispositions of things as revealed by changes in entropy. We might measure the entropy change associated with a given change through the notion of entropy as non-dimensional energy change. We might also measure through counting things and possible arrangements by which we understand a universal relation to exist between the hidden energy and the arrangement or number of material things.

Changes in entropy can be established by measuring changes in the dispositions or capacities of things which we general term energy, the most intrinsic to a population of particles being the internal 
energy or average kinetic energy of particles. Since energy itself is metaphysical and we only measure indirectly, through the effects of these capacities on the spatial presentation of matter, it can be argued that energy is intangible. Our measurement of entropy changes through non-dimensional energy changes that are inferred from macroscopic properties of a system can be related to the measurement of entropy by statistical mechanical means. The statistics proceeds by observing conserving of mass and energy, the energy being distributed such that certain rules are obeyed with respect to spreading out the energy of the system.

\subsection{Some Challenges to the Views Presented Herein}

We have noted that entropy changes are related to non-equilibrium interactions of subsystems which had hitherto been in equilibrium or the progress of a non-equilibrium system after perturbation toward a new equilibrium state. Relating time to the associated entropy changes means difficulty in time measurement at equilibrium. That is, if thermodynamic driving forces that lead to entropy generation are absent, it would then be difficult to tell the passage of time on account of the constant entropy measured in the system or systems. For instance, one would have difficulty in measuring time using two systems in rectilinear motion with constant but different velocities. Their separation in space grows and can become a basis of time measurement following current practices but there is no formal way of casting this growing separation between them into entropy change to accord with the view presented here.

One who is committed to defending the view advanced here would first argue for the need to generalize entropy production as involving forces other than thermal. Such is the case when one defends the directionality of Fickian diffusion in which heat transfer is not involved. The entropy grows on account of differences in chemical potentials being minimized. Entropy changes must be made to capture all types of non-equilirbium motions or changes. Such a defender might proceed to argue against the possibility of any truly uniform rectilinear motion on account of ever present gravitational interactions which cannot be screened out of a practical system. If this is admitted, then the problem falls to poor conceptualization of the operative acceleration. Once acceleration is operative, then there must be a resultant force and associated work that enables non-equilibrium change. If Gibbs' idea that all dynamic problems are to be treated such that the change in energy is related to the sum of the product of driving forces and changes in their respective coordinates [17], then admitting existence of acceleration in any system, also admits of driving forces, changes in coordinates and energy of the system, such that a redefined entropy to read as non-dimensional measure of the change of a conserved quantity would secure the relation between entropy measure and time duration.

A second challenge that might be levelled against the supposed relation between entropy change and time would be the notion of time reversibility that is thought to be anchored in the equations of classical mechanics such as Newton's second axiom. This view was a stumbling block to the reception of Boltzmann's seminal work on statistical mechanics. The directedness of time that coincides with the natural decrease of Boltzmann's H-function, and therefore entropy increase, seems to be at odds with observed time reversal in classical mechanics. This difficulty is also a stumbling block for Prigogine whose discovery of dissipative structures, notwithstanding, still seems to fail to resolve the time reversibility question. I think that the problem lies in a misunderstanding of the properties of mathematics and properties of the physical relations or physical quantities whose dynamics is described using mathematics.

There is a distinction between the properties of mathematical functions or operations and the physics that is mathematically represented. Suppose we represent the temporal evolution of the population of a given city using a third-order polynomial and we seek to find the year in which the population assumed a certain number, we might solve this and obtain three real roots or one real root and two imaginary roots. We reject the imaginary roots on the strength of empirical evidence against a physical population being imaginary. The same goes for negative values of time, if the time is counted from the origin of the population. The situation changes if we had chosen to approximate the 
population using an exponential function. In this case, the time corresponding to a given population is unique and requires no further reasoning to interpret it. This subtle distinction between the properties of mathematics and of physics needs to be carried over to the equations of classical mechanics.

From Newton's second law, $\vec{F}=m \vec{a}=\frac{d \vec{P}}{d t}$, it requires that the direction of acceleration aligns with the direction of the resultant force. The resultant force might be likened to the driving force in a thermodynamic system that brings about natural change and entropy increase. The supposed time reversal is in essence a physical action on the system from without that changes the direction of the resultant forces so as to produce the seemingly unusual property of time reversal for a process described using classical mechanics. A change of sign is a change of the driving forces and must be properly factored in. It must therefore be argued that the requirement that accelerations or retardations must align with the direction of resultant forces on the classical systems is an affirmation of the directedness of natural processes such as the flow of heat from hot to cold regions, coinciding with an increase in entropy. It must only be ascertained how a more general entropy change that captures the non-equilibrium of Newton's Second Law might be defined. In more general terms one can speak of the extremization of a given conserved property of dynamic system. The motion toward minimum energy is a universal directedness that could be captured with a properly defined entropy and in the absence of external driving forces, the entropy change could serve as a clock for measuring time.

\section{Conclusions}

This paper explored the possibility of a relationship between measurement of entropy change and durations of time. Taking entropy to be a measure of non-dimensional energy of a system resulting from a driving force such as temperature gradients, a relationship can be shown. The simple case of steady transfer between two systems of infinite heat capacities shows how and approximately linear relationship obtains. This points to changes in the configuration of physical things, and by extension in the intrinsic energy of a system, to be more fundamental than time. Time plays the role of a mental awareness of relative change, where memory of the initial state is recollected and compared with the present state of affairs. As long as there is no further energy injection or withdrawal from an isolated system, a clock based on entropy measurement can be constructed. The entropy change can be ascertained through indirect energy measurement using macroscopic variables or the configurations of microscopic particles which correlate to the quantity and spread of energy in the system.

Conflicts of Interest: The authors declare no conflict of interest.

\section{References}

1. Prigogine, I.; Stengers, I. The End of Certainty; Simon and Schuster: New York, NY, USA, 1997.

2. Prigogine, I. Time, structure, and fluctuations. Science 1978, 201, 777-785.

3. Bowin, J. Aristotle on the Order and Direction of Time. Apeiron 2009, 42, 33-62.

4. Clausius, R. Über verschiedene für die Anwendung bequeme Formen der Hauptgleichungen der mechanischen Wärmetheorie. Ann. Phys. 1865, 201, 353-400.

5. Uffink, J. Bluff your way in the second law of thermodynamics. Stud. Hist. Philos. Sci. 2001, 32, 305-394.

6. Akih-Kumgeh, B. Toward improved understanding of the physical meaning of entropy in classical thermodynamics. Entropy 2016, 18, 270.

7. Kant, I. Critique of Pure Reason. Translated by Norman Kemp Smith; London Macmillan: London, UK, 1934.

8. Swinburne, R. Space and Time; Springer: Berlin/Heidelberg, Germany, 1968.

9. Reichenbach, H. The Philosophy of Space and Time; Courier Corporation: North Chelmsford, MA, USA, 2012.

10. Le Poidevin, R. The Experience and Perception of Time. In The Stanford Encyclopedia of Philosophy, Summer 2015 ed.; Zalta, E.N., Ed.; Metaphysics Research Lab., Stanford University: Stanford, CA, USA, 2015.

11. McTaggart, J.E. The unreality of time. Mind 1908, 457-474. 
12. Rynasiewicz, R. Newton's Views on Space, Time, and Motion. In The Stanford Encyclopedia of Philosophy, Summer 2014 ed.; Zalta, E.N., Ed.; Metaphysics Research Lab., Stanford University: Stanford, CA, USA, 2014.

13. Essen, L.; Parry, J. An atomic standard of frequency and time interval: A caesium resonator. Nature 1955, 176, 280-282.

14. Kondepudi, D.; Prigogine, I. Modern Thermodynamics: From Heat Engines to Dissipative Structures; John Wiley \& Sons: Hoboken, NJ, USA, 2014.

15. Velasco, R.M.; Scherer García-Colín, L.; Uribe, F.J. Entropy Production: Its Role in Non-Equilibrium Thermodynamics. Entropy 2011, 13, 82-116.

16. Onsager, L. Reciprocal relations in irreversible processes. I. Phys. Rev. 1931, 37, 405.

17. Gibbs, J.W. On the fundamental formulae of dynamics. Am. J. Math. 1879, 2, 49-64.

(C) 2018 by the author. Licensee MDPI, Basel, Switzerland. This article is an open access article distributed under the terms and conditions of the Creative Commons Attribution (CC BY) license (http://creativecommons.org/licenses/by/4.0/). 